\title{
Características buco-dentárias de pacientes leucêmicos: uma revisão sistemática
}

\author{
Oral-dentalcharacteristics of leukemic patients: a systematic review
}

Características oral-dentales de pacientes leucêmicos: uma revisíon sistemática

Glaudemir Santos Leite1, Maria José Pergentino Batista Bezerra1, Virginia Karla Pinheiro de Queiroz $^{2}$, David Jorge Pereira Alves², Lídia Pinheiro da Nóbrega², Welma Emidio da Silva*.

\section{RESUMO}

Objetivo: Analisar as características buco-dentárias de portadores de leucemia, identificando os fatores casuísticos. Métodos: Realizou-se de uma revisão sistemática da literatura, onde as bases de dados Biblioteca Científica Eletrônica Online (Scielo), Literatura Latino-Americana e do Caribe em Ciências da Saúde (Lilacs) e Sistema Online de Busca de Análise de Literatura Médica (Medline) foram utilizadas para a busca de artigos científicos. Foram utilizados como critérios de inclusão: tipo de estudo, ano de publicação, idioma e disponibilidade de acesso. Foram excluídos os relatos de casos, as revisões de literatura, as duplicatas e os que não atenderam os objetivos da revisão. Resultados: Dos 351 estudos encontrados nas referidas bases de dados, apenas 17 atenderam aos critérios de inclusão. Várias alterações histopatológicas foram registradas nas cavidades orais dos participantes com leucemia. Mucosite, gengivite, petéquias, equimose, sangramento, ulcerações, palidez da mucosa, xerostomia, hiperplasia gengival, inflamação, alteração da microbiota e composição salivar foram as alterações encontradas pelos estudos. Tais alterações são exacerbadas pelo tratamento antineoplásico, condições hematológicas e higiene oral do paciente. Considerações Finais: Pacientes com leucemia normalmente apresentam alterações histopatológicas na cavidade oral e vários fatores influenciam na frequência, grau de severidade e tipo de lesão encontrada.

Palavras-chave: Manifestações bucais, Oncologia, Quimioterapia.

\begin{abstract}
Objective: To analyze the oral and dental characteristics ofleukemia patients, identifying the casuistic factors. Methods: A systematic literature review was carried out, where the Scientific Eletronic Library Online (Scielo), Latin American and Caribbean Literature in Health Science (Lilacs) and Medical Literature Analysis and Retrieval System Online (Medline) databases were used to search scientific articles. Inclusion criteria were used: type of study, year of publication, language and availability access. Case reports, literature reviews, duplicates and those that did not meet the objectives of the review were excluded. Results: Of the 351 studies found in referred databases, only 17 met the inclusion criteria. Several histopathological changes were recorded in oral cavities of participants with leukemia. Mucositis, gingivitis, petechiae, ecchymosis, bleeding, ulcerations, mucous pallor, xerostomia, gingival hyperplasia, inflammation, alteration of microbiota and salivary composition were alterations found by the studies. Such changes are exacerbated by antineoplastic treatment, hematological conditions and oral hygiene of patient. Final Considerations: Patients with leukemia usually present histopathological changes in oral cavity and several factors influence the frequency, degree severity and type of lesion found.
\end{abstract}

Keywords: Oral manifestations, Oncology, Chemotherapy.

${ }^{1}$ Centro Universitário Maurício de Nassau (UNINASSAU), Caruaru - PE.

${ }^{2}$ Faculdade de Integração do Sertão (FIS), Serra Talhada - PE. *E-mail: welmaesilva@gmail.com 


\section{RESUMEN}

Objetivo: Analizar las características oral-dentales en pacientes con leucemia, identificando los factores casuísticos. Métodos: Se realizó una revisión sistemática de la literatura, donde se utilizaron las bases de datos de la Biblioteca Científica Eletrônica en Línea (Scielo), la Literatura Latinoamericana y Caribeña em Ciencias de la Salud (Lilacs) y el Sistema de Búsqueda de Análises de Literatura Médica en Línea (Medline) para buscar artículos científicos. Se utilizaron criterios de inclusión: tipo de estudio, año de publicación, idioma y disponibilidad de acceso. Se excluyeron los informes de casos, revisiones de literatura, duplicados y aquellos que no cumplieron con los objetivos de la revisión. Resultados: De los 351 estudios encontrados en las bases de datos referidas, solo 17 cumplieron los criterios de inclusión. Se registraron varios cambios histopatológicos en las cavidades orales de los participantes con leucemia. Mucositis, gingivitis, petequias, equimosis, sangrado, ulceraciones, palidez mucosa, xerostomía, hiperplasia gingival, inflamación, alteración de la microbiota y composición salival fueron las alteraciones encontradas por los estudios. Dichos cambios se ven exacerbados por el tratamiento antineoplásico, las condiciones hematológicas y la higiene bucal del paciente. Consideraciones Finales: Los pacientes con leucemia generalmente presentan cambios histopatológicos en la cavidad oral y varios factores influyen en la frecuencia, el grado de gravedad y el tipo de lesión encontrada.

Palabras clave: Manifestaciones orales, Oncología, Quimioterapia.

\section{INTRODUÇÃO}

As leucemias são doenças neoplásicas que alteram as células hematopoiéticas e são um tipo de câncer muito comum tanto em crianças como em adultos (MCCORD C e JOHNSON L, 2017). Sua principal característica é a proliferação desregulada de células jovens da medula óssea, com alterações nos mecanismos de diferenciação e apoptose, que passam a substituir as células sanguíneas normais de forma afuncional (FINOTTI ACF, et al., 2006; VALÉRA MC, et al., 2014).

$\mathrm{Na}$ maioria dos casos, as células leucêmicas extravasam para o sangue, onde podem ser vistas em grande número, e à medida que as células neoplásicas se disseminam pela circulação diversos órgãos e tecidos, tais como o fígado, baço e linfonodos são afetados (GRAZIELE C, et al., 2006). Desta forma, as manifestações clínicas dessa doença podem ocorrer em todos os órgãos e tecidos irrigados e nutridos pela corrente sanguínea, inclusive os tecidos bucais (COSTA SS, et al., 2011).

De acordo com a linhagem celular imatura (blástica) de origem, as leucemias podem originar distúrbios mieloproliferativos (proliferação neoplásica de granulócitos, monócitos, eritrócitos, megacariócitos e mastócitos) como linfoproliferativos (que se limitam à proliferação neoplásica de linfócitos). Ainda, de acordo com o curso clínico, a doença pode se classificar como aguda ou crônica (COSTA SS, et al., 2011; MCCORD C e JOHNSON L, 2017).

As leucemias são grupos heterogêneos de neoplasias hematológicas, sendo o diagnóstico precoce, juntamente com tratamentos eficazes, de extrema importância para o prognóstico e qualidade de vida do paciente (JESUS LG, et al., 2016). Leucemia aguda implica numa apresentação clínica relativamente breve, comportamento biológico agressivo, quantidades variáveis de células imaturas no sangue e medula óssea, curta expectativa de sobrevida e maior incidência em crianças. As leucemias crônicas, sugerem o curso clínico prolongado, comportamento biológico menos agressivo, aumento no número de células bem diferenciadas, expectativa de vida mais prolongada e maior incidência nos adultos (NELSON RW e COUTO CG, 2015).

Independente da classificação, a leucemia apresenta as primeiras manifestações na mucosa bucal (HESPANHOL FL, et al., 2010; COSTA SS, et al., 2011). As manifestações orais podem ser graves, levando a complicações sistêmicas importantes que podem aumentar o tempo de internação hospitalar e os custos do tratamento, bem como a interrupção do tratamento oncológico, já que esse fator reduz a sobrevida do paciente e o predispõe a inúmeras infecções (JESUS LG, et al., 2016). 
Dessa forma, o reconhecimento das lesões na cavidade oral em pessoas com leucemia, bem como os fatores que levaram ao surgimento das mesmas, permite que os cirurgiões-dentistas atuem na prevenção e no tratamento adequado, melhorando a saúde bucal dos indivíduos acometidos pela leucemia (HESPANHOL FL, et al., 2010; MORAIS EF, et al., 2014).

Diante do exposto, este estudo tem o objetivo de fazer uma revisão sistemática da literatura sobre alterações histopatológicas da cavidade oral em portadores de leucemia, identificando os fatores casuísticos.

\section{MÉTODOS}

Realizou-se uma busca sistemática de artigos científicos que abordam a relação entre leucemia e manifestações orais. A pesquisa se realizou em fevereiro de 2020 e a busca por artigos foi executada nas bases de dados eletrônicas Scielo, Lilacs e Medline, utilizando os Descritores em Ciências da Saúde (DeCS): "Manifestações bucais", "Oncologia" e "Quimioterapia", assim como seus sinônimos e correspondentes na língua inglesa. Para complementar a busca, foi realizada uma busca manual em listas de referências de artigos relevantes para esta revisão.

Utilizou-se como critério de inclusão: tipo de estudo (estudos clínicos e experimentais que avaliaram manifestações orais em portadores de leucemia), ano de publicação (publicados entre 2009 e 2020), idioma (português, espanhol e inglês) e disponibilidade de acesso (o texto completo tinha que estar disponível na íntegra). Foram excluídos os relatos de caso, revisões de literatura, estudos publicados em duplicatas nas bases de dados e os que não atendessem aos objetivos da pesquisa. Uma primeira etapa de seleção foi feita a partir da avaliação dos títulos e resumos. Posteriormente, os estudos cujos resumos apresentaram dados julgados interessantes para análise, foram obtidos na íntegra. Os artigos lidos por completo e que se enquadraram nos critérios de inclusão foram eleitos para compor a revisão.

\section{RESULTADOS}

A pesquisa resultou em 351 artigos distribuídos nos bancos de dados avaliados. Após a análise dos títulos e resumos, 321 estudos foram excluídos e 30 foram lidos na íntegra. Destes, apenas 18 preencheram todos os critérios de inclusão (Figura 1). Os estudos elegidos para compor esta revisão estão detalhados na tabela abaixo (Tabela 1).

Figura 1 - Fluxograma da estratégia de busca para identificar estudos elegíveis.

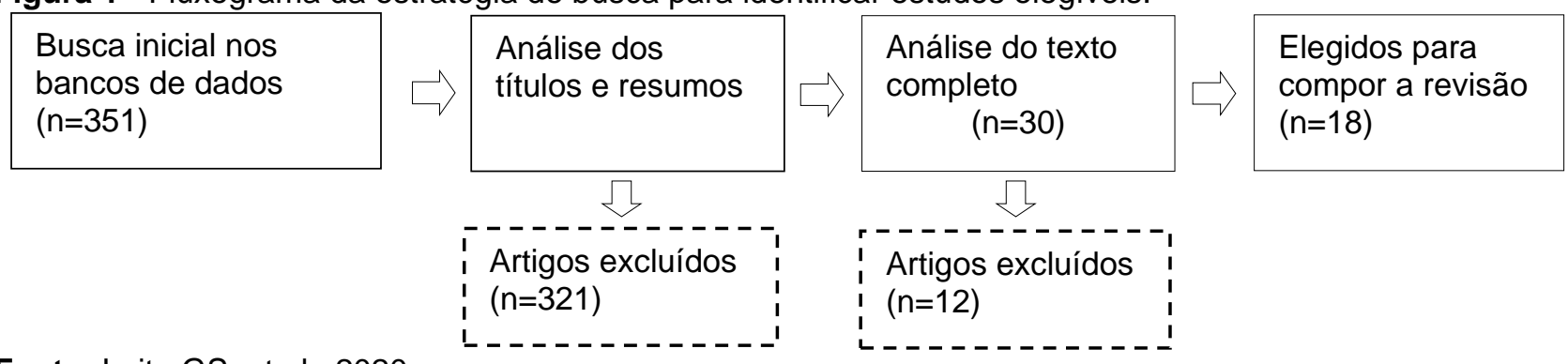

Fonte: Leite GS, et al., 2020

Todos os estudos elegidos para compor a revisão avaliaram pacientes com Leucemia em estágio agudo; apenas dois incluíram em sua amostra participantes em estágio crônico. Quanto a origem da linhagem celular, doze estudos analisaram participantes com leucemia de origem linfóide, cinco apresentavam uma amostra mista (linfoide e mieloide) e apenas um apresentava amostra com participantes com leucemia mieloide aguda. Apesar de a amostra de Thomaz EBA, et al. (2013) ter sido mista, a maioria (95\%) dos participantes apresentavam leucemia de origem linfoide (Quadro 1).

Quanto o perfil das amostras, os estudos apresentaram um número de participantes que oscilou entre 17 e 156 pessoas, com idade que variou dos dez meses aos 80 anos. No entanto, a maioria utilizou amostra composta por adolescentes e/ou crianças. Apenas um utilizou uma amostra composta apenas por adultos e três utilizaram amostra heterogênea quanto a idade. 
Revista Eletrônica Acervo Saúde / Electronic Journal Collection Health | ISSN 2178-2091

Quadro 1 - Sumarização de dados dos estudos elegidos.

\begin{tabular}{|c|c|c|c|c|}
\hline Estudo & Tipo de leucemia & $\mathbf{n}$ & Idade & Resultado principal \\
\hline $\begin{array}{l}\text { ANGST PDM, et al., } \\
2011 .\end{array}$ & $\begin{array}{l}\text { LMA, LMC, } \\
\text { LLA e LLC }\end{array}$ & $37 \mathrm{~L}$ & $15-80$ anos & $\begin{array}{c}\text { Não houve correlação significativa entre a baixa contagem de plaquetas } \\
\text { e a prevalência de sangramento gengival e periodontal. }\end{array}$ \\
\hline ARUL ASKJ, et al., 2012. & LMA e LLA & $50 \mathrm{~L}$ & $11-55$ anos & Verificou-se que a infiltração gengival leucêmica é mais comum na LLA. \\
\hline ASHOK L, et al. (2010) & $\begin{array}{l}\text { LMA, LMC, } \\
\text { LLA e LLC }\end{array}$ & $\begin{array}{l}30 \mathrm{~L} \\
25 \mathrm{C}\end{array}$ & 03-80 anos & $\begin{array}{l}\text { Detectou-se mudanças significativas na composição salivar de } \\
\text { pacientes leucêmicos e manifestações orais na maioria destes. }\end{array}$ \\
\hline $\begin{array}{l}\text { AZHER U e SHIGGAON } \\
\text { N, } 2013 .\end{array}$ & LLA & $94 \mathrm{~L}$ & 02-14 anos & $\begin{array}{c}\text { Houve diferença significativa do índice gengival, úlceras e dificuldade de } \\
\text { deglutição entre as fases do tratamento. }\end{array}$ \\
\hline $\begin{array}{l}\text { CORONEL JP, et al., } \\
\quad 2016 .\end{array}$ & LLA & $\begin{array}{l}32 \mathrm{~L} \\
32 \mathrm{C}\end{array}$ & $01-13$ anos & $\begin{array}{l}\text { Crianças com LLA apresentaram maior frequência e vulnerabilidade de } \\
\text { sofrer lesões estomatológicas que crianças saudáveis. }\end{array}$ \\
\hline $\begin{array}{l}\text { GHOLIZADEH N, et al., } \\
2016 .\end{array}$ & LLA & $90 \mathrm{~L}$ & 05-18 anos & $\begin{array}{c}\text { A palifermina diminuiu incidência e gravidade da mucosite induzida pela } \\
\text { quimioterapia. }\end{array}$ \\
\hline HEGDE AM, et al., 2011. & LLA & $\begin{array}{l}90 \mathrm{~L} \\
30 \mathrm{C}\end{array}$ & 04-10 anos & $\begin{array}{l}\text { Crianças leucêmicas apresentaram deterioração da saúde bucal, do } \\
\text { estado gengival e aumento da experiência de cárie. Também houve } \\
\text { redução do fluxo e pH salivar e alteração dos níveis de antioxidantes } \\
\text { salivares. }\end{array}$ \\
\hline JOSHI S, et al., 2013. & LLA & $\begin{array}{l}90 \mathrm{~L} \\
30 \mathrm{C}\end{array}$ & 04-10 anos & $\begin{array}{l}\text { Houve relação linear entre os níveis de ácido siálico e cárie e relação } \\
\text { inversa entre fluxo salivar e cárie dentária em crianças leucêmicas. }\end{array}$ \\
\hline $\begin{array}{l}\text { JUÁREZ-LÓPEZ MLA, et } \\
\text { al., } 2018 .\end{array}$ & LLA & $103 \mathrm{~L}$ & 03-15 anos & $\begin{array}{l}\text { A frequência e gravidade das doenças bucais foram elevadas e } \\
\text { associadas à fase de indução da quimioterapia, alterando a nutrição e a } \\
\text { saúde geral das crianças. }\end{array}$ \\
\hline $\begin{array}{l}\text { OLIVEIRA MC, et al., } \\
2016 .\end{array}$ & LLA e LMA & $36 \mathrm{~L}$ & 10 meses -15 anos & $\begin{array}{l}\text { Anormalidades orais foram comuns em crianças que receberam } \\
\text { quimioterapia, sendo a mucosite a mais prevalente. A mucosite não } \\
\text { estava associada à higiene oral. }\end{array}$ \\
\hline
\end{tabular}

REAS / EJCH | Vol.12(11) | e4480 | DOI: https://doi.org/10.25248/reas.e4480.2020 Página 4 de 11 
Revista Eletrônica Acervo Saúde / Electronic Journal Collection Health | ISSN 2178-2091

\begin{tabular}{|c|c|c|c|c|}
\hline Estudo & Tipo de leucemia & $\mathbf{n}$ & Idade & Resultado principal \\
\hline PELS EJ, et al., 2017. & LLA & $\begin{array}{l}78 \mathrm{~L} \\
78 \mathrm{C}\end{array}$ & 02-18 anos & $\begin{array}{l}\text { Houve diminuição significativa nas concentrações salivares de } \lg A \text {, } \lg G \\
\text { e } \operatorname{lgM} \text { em crianças com leucemia. }\end{array}$ \\
\hline $\begin{array}{l}\text { PONCE-TORRES E, et } \\
\text { al., } 2010 .\end{array}$ & LLA & $49 \mathrm{~L}$ & 02-14 anos & $\begin{array}{c}\text { O tipo de leucemia, sexo e fase da quimioterapia foram aparentemente } \\
\text { associados à presença de candidíase, gengivite e periodontite, podendo } \\
\text { ser considerados fatores de risco para o desenvolvimento de } \\
\text { manifestações orais. }\end{array}$ \\
\hline $\begin{array}{l}\text { SHANKARAPILLAI R, et } \\
\text { al., } 2010 .\end{array}$ & LMA & $73 \mathrm{~L}$ & 18-27 anos & $\begin{array}{l}\text { Houve associação significativa da má higiene bucal com risco para } \\
\text { crescimento gengival, gengivite e doença periodontal destrutiva em } \\
\text { pacientes com LMA. }\end{array}$ \\
\hline SOARES AF, et al., 2011. & LLA & $17 \mathrm{~L}$ & 02-12 anos & $\begin{array}{l}\text { O uso de gluconato de clorexidina a } 0,12 \% \text { reduziu a frequência de } \\
\text { mucosite oral e patógenos orais em crianças com LLA. }\end{array}$ \\
\hline $\begin{array}{l}\text { THOMAZ EBA, et al., } \\
2013 .\end{array}$ & LLA e LMA & $\begin{array}{l}20 L \\
22 C\end{array}$ & 03-15 anos & $\begin{array}{l}\text { As condições clínicas e imunológicas da cavidade oral de crianças e } \\
\text { adolescentes submetidos à quimioterapia apresentam evolução } \\
\text { desfavorável com redução de } \lg A \text { e a presença de gengivite e mucosite. }\end{array}$ \\
\hline $\begin{array}{l}\text { TRINDADE AKF, et al., } \\
2009 .\end{array}$ & LLA & $16 \mathrm{~L}$ & 03-17 anos & $\begin{array}{l}\text { Pacientes com melhores condições de saúde oral e higiene e higiene } \\
\text { oral satisfatória exibem menos manifestações e estas têm curso clínico } \\
\text { mais rápido. }\end{array}$ \\
\hline $\begin{array}{l}\text { VASCONCELOS NPS, et } \\
\text { al., } 2009 .\end{array}$ & LLA & $\begin{array}{l}46 \mathrm{~L} \\
46 \mathrm{C}\end{array}$ & 04-12 anos & $\begin{array}{l}\text { Houve uma diferença significativa da idade cronológica e odontológica } \\
\text { nos pacientes submetidos à terapia antineoplásica. }\end{array}$ \\
\hline WANG Y, et al., 2014. & LLA & $\begin{array}{r}13 L \\
12 C\end{array}$ & 02-14 anos & $\begin{array}{l}\text { Houve desequilíbrio da microbiota bucal entre crianças leucêmicas e } \\
\text { saudáveis. }\end{array}$ \\
\hline
\end{tabular}

REAS / EJCH | Vol.12(11) | e4480 | DOI: https://doi.org/10.25248/reas.e4480.2020 Página 5 de 11 
Quando analisado o período de realização do estudo, se verificou que cinco foram realizados com participantes antes ou sem tratamento; seis com participantes durante o tratamento; seis com participantes antes e durante/após o tratamento e apenas um utilizou participantes que já haviam finalizado o tratamento há pelo menos 12 meses (Quadro 2).

Quadro 2 - Análise do período de realização e tipo de tratamento dos participantes dos estudos.

\begin{tabular}{|c|c|c|c|}
\hline Estudo & $\begin{array}{l}\text { Período de } \\
\text { realização do estudo }\end{array}$ & $\begin{array}{l}\text { Tipo de } \\
\text { tratamento }\end{array}$ & Fase do tratamento \\
\hline $\begin{array}{c}\text { ANGST PDM, et al., } \\
2011 \\
\end{array}$ & DT & Não informado & Não especificou \\
\hline ARUL ASKJ, et al., 2012 & AT & Não informado & Não informado \\
\hline ASHOK L, et al., 2010. & ST & Não informado & Não informado \\
\hline $\begin{array}{c}\text { AZHER U e SHIGGAON } \\
\text { N, } 2013 .\end{array}$ & DT & $\begin{array}{c}\text { Q }(64 \%) \text { e } R \\
(34 \%)\end{array}$ & Indução e Manutenção \\
\hline $\begin{array}{l}\text { CORONEL JP, et al., } \\
2016 .\end{array}$ & AT & Não informado & Não informado \\
\hline $\begin{array}{l}\text { GHOLIZADEH N, et al., } \\
2016 .\end{array}$ & $\begin{array}{l}\text { AT e PT (1 e } 2 \\
\text { semanas) }\end{array}$ & Q & Indução e Intensificação \\
\hline HEGDE AM, et al., 2011. & AT e DT & $Q$ & Não informado \\
\hline JOSHI S, et al., 2013. & AT e DT & $Q$ & Não informado \\
\hline $\begin{array}{l}\text { JUÁREZ-LÓPEZ MLA, et } \\
\text { al., } 2018 .\end{array}$ & DT & Q & Indução \\
\hline $\begin{array}{l}\text { OLIVEIRA MC, et al., } \\
2016 .\end{array}$ & DT & $\begin{array}{c}Q(86,1 \%) e \\
Q+R(13,9 \%)\end{array}$ & $\begin{array}{l}\text { Indução, Manutenção da } \\
\text { remissão e Manutenção pós- } \\
\text { recaída }\end{array}$ \\
\hline PELS EJ, et al., 2017. & AT e DT & $Q$ & Não informado \\
\hline $\begin{array}{l}\text { PONCE-TORRES E, } \\
2010 .\end{array}$ & DT & Q & $\begin{array}{c}\text { Manutenção, indução de } \\
\text { consolidação e consolidação. }\end{array}$ \\
\hline $\begin{array}{l}\text { SHANKARAPILLAI R, et } \\
\text { al., } 2010 .\end{array}$ & AT & Q & Não informado \\
\hline $\begin{array}{c}\text { SOARES AF, et al., } \\
2011 .\end{array}$ & DT & Q & $\begin{array}{c}\text { Indução, consolidação, } \\
\text { intensificação, manutenção }\end{array}$ \\
\hline $\begin{array}{l}\text { THOMAZ EBA, et al., } \\
2013 .\end{array}$ & $\begin{array}{c}\text { AT/IT e } \\
\text { PT (6 meses) }\end{array}$ & Q & Não informado \\
\hline $\begin{array}{l}\text { TRINDADE AKF, et al., } \\
2009 .\end{array}$ & DT & Q & $\begin{array}{l}\text { Indução, Manutenção, } \\
\text { Intensificação e consolidação }\end{array}$ \\
\hline $\begin{array}{c}\text { VASCONCELOS NPS, } \\
\text { et al., } 2009 .\end{array}$ & PT ( $\geq 12$ meses) & $\begin{array}{c}Q(67,39 \%) e \\
Q+R(32,61 \%)\end{array}$ & Não informado \\
\hline WANG Y, et al., 2014. & AT & Não informado & Não informado \\
\hline
\end{tabular}

Legenda: AT- antes do tratamento. DT - duramte o tratamento. PT- pós-tratamento. IT- início do tratamento. Q- quimioterapia. R- radioterapia.

Fonte: Leite GS, et al., 2020.

Dos estudos que informaram a modalidade de tratamento dos participantes, apenas três utilizaram amostra composta por participantes que receberam quimioterapia (maioria) e radioterapia, os demais utilizaram apenas pacientes que receberam apenas quimioterapia. E, apesar de dez artigos terem citado as drogas ou protocolos de tratamento utilizadas durante a quimioterapia, nenhum estabeleceu correlação clara entre complicações orais e tipo de droga utilizada. Dos estudos que avaliaram as condições orais durante $o$ tratamento, apenas três teve a relação entre as complicações orais observadas e a fase da quimioterapia que o participante se encontrava estabelecida. 
Quadro 3 - Alterações orais relatadas pelos estudos elegidos para compor a revisão.

\begin{tabular}{|c|c|}
\hline Estudo & Manifestações orais \\
\hline ANGST PDM, et al., 2011. & $\begin{array}{c}\text { Inflamação subgengival; inflamação leve na gengiva marginal; perda } \\
\text { moderada de inserção. }\end{array}$ \\
\hline ARUL ASKJ, et al., 2012, & $\begin{array}{l}\text { Infiltração de células leucêmicas no tecido gengival (mais comum em LLA); } \\
\text { sangramento e aumento gengival. }\end{array}$ \\
\hline ASHOK L, et al., 2010. & $\begin{array}{l}\text { Palidez; gengivite; aumento gengival; petéquias e equimoses; manifestações } \\
\text { radiográficas nas mandíbulas (rarefação óssea generalizada, } \\
\text { adelgaçamento da lâmina dura, reabsorção óssea da crista alveolar e lesões } \\
\text { periapicais); alteração na composição salivar. }\end{array}$ \\
\hline $\begin{array}{l}\text { AZHER U e SHIGGAON } \\
\text { N, } 2013 .\end{array}$ & $\begin{array}{l}\text { Úlceras nos lábios e na mucosa oral; inflamação gengival, principalmente } \\
\text { nas fases de manutenção }(Q) \text { e indução }(R) \text {; mucosite; maior índice de cárie } \\
\text { nas fases de manutenção }(Q) \text { e indução (radioterapia). }\end{array}$ \\
\hline $\begin{array}{l}\text { CORONEL JP, et al., } \\
2016 .\end{array}$ & $\begin{array}{l}\text { Úlcera; sangramento espontâneo; equimoses; palidez da mucosa; gânglios } \\
\text { linfáticos palpáveis. }\end{array}$ \\
\hline $\begin{array}{l}\text { GHOLIZADEH N, et al., } \\
2016 .\end{array}$ & Mucosite. \\
\hline 2011. & $\begin{array}{l}\text { Fluxo e pH salivar reduzidos; alteração dos níveis de a } \\
\text { redução da higiene oral; deterioração da saúde gengival }\end{array}$ \\
\hline JOSHI S, et al., 2013. & $\begin{array}{l}\text { Aumento de cárie; redução de fluxo salivar; aumento do valor médio do } \\
\text { índice de CPOD com o tempo de quimioterapia. }\end{array}$ \\
\hline $\begin{array}{r}\text { JUÁREZ-LÓPI } \\
\text { al., } 20\end{array}$ & ceras. \\
\hline $\begin{array}{l}\text { OLIVEIRA MC, et al., } \\
2016 .\end{array}$ & $\begin{array}{r}\text { Mucosite; sangramento gengival; membranas mu } \\
\text { membranas mucosas pálidas e }\end{array}$ \\
\hline PELS EJ, et al., 2017. & $\begin{array}{l}\text { Eritrema; mucosa pseudomembranosa; úlceras com eritrema; necrose; } \\
\text { infecção fúngica. }\end{array}$ \\
\hline $\begin{array}{l}\text { PONCE-TORRES E, et } \\
\text { al., } 2010 .\end{array}$ & $\begin{array}{c}\text { Gengivite, cárie, mucosite, periodontite, queilite, herpes recorrente, } \\
\text { candidíase, gengivostomatite herpética primária, lábios secos, palidez da } \\
\text { mucosa oral, hipoplasia do esmalte, petéquias, equimoses e úlceras } \\
\text { induzidas. }\end{array}$ \\
\hline $\begin{array}{l}\text { SHANKARAPILLAI R, et } \\
\text { al., } 2010 .\end{array}$ & Aumento gengival; perda do suporte periodontal; inflamação gengival. \\
\hline SOARES AF, et al., 2011. & Mucosite; infe \\
\hline $\begin{array}{l}\text { THOMAZ EBA, et al., } \\
2013 .\end{array}$ & $\begin{array}{l}\text { Gengivite; mucosite; aumento do índice médio de CPOD; experiência de } \\
\text { cárie moderada a alta; deterioração da saúde gengival; redução de lgA. }\end{array}$ \\
\hline $\begin{array}{l}\text { TRINDADE AKF, et al., } \\
2009 .\end{array}$ & $\begin{array}{l}\text { Petéquia; mucosite em grau I, II e III; lígua despapilada; hiperplasia e } \\
\text { sangramento gengival; palidez da mucosa; língua saburrosa; cárie; mácula } \\
\text { melanótica; anquiloglossia. }\end{array}$ \\
\hline $\begin{array}{l}\text { VASCONCELOS NPS, et } \\
\text { al., } 2009 .\end{array}$ & o desenvolvimento radicular. \\
\hline WANG Y, et al., 2014. & robiota oral. \\
\hline
\end{tabular}

Legenda: Q- quimioterapia. R- radioterapia. pH- potencial hidrogeniônico. LLA-leucemia linfoblástica aguda. Fonte: Leite GS, et al., 2020.

As alterações orais em pessoas com leucemia relatadas nos estudos estão descritas na tabela acima (Quadro 3). Apenas quatro estudos, especificaram quais lesões eram encontradas antes, durante e/ou após o tratamento antineoplásico. Apesar de Agnst, et al. (2011) e Pels EJ, et al. (2017) terem relatado os tipos de lesões observadas na cavidade oral dos participantes, não especificaram os períodos de surgimento das lesões. Apenas um estudo utilizou a presença de mucosite oral ou ouras lesões orais antes da quimioterapia como critério de exclusão (GHOLIZADEH N, et al., 2016).

Quanto ao perfil hematológico, apenas três estudos realizaram análise comparativa dos elementos sanguíneos dos participantes com os resultados encontrados (TRINDADE AKF, et al., 2009; ANGST PDM, et al., 2011; PELS EJ, et al., 2017). A relação da higiene oral com as alterações bucais observadas foi realizada em quatro estudos (SHANKARAPILLAI R, et al., 2010; HEGDE AM, et al., 2011; AZHER U e SHIGGAON N, 2013; OLIVEIRA MC, et al., 2016). 


\section{DISCUSSÃO}

Leucemia é um termo utilizado para designar um grupo de doenças neoplásicas malignas dos tecidos formadores de sangue, caracterizadas pela produção de um grande número de células sanguíneas imaturas (AZHER U e SHIGGAON N, 2013). Por afetar diferentes tipos de leucócitos e apresentar duração variável, ela pode ser classificada baseado na célula de origem (linfóide ou mielóide) ou com base na velocidade em que a doença evolui (aguda ou crônica) (JAVED F, et al., 2012; VALÉRA MC, et al., 2014).

Entretanto, independentemete da forma que a leucemia se apresente, os primeiros sinais podem surgir na cavidade oral e podem ser visualizadas e reconhecidas pelo cirurgião-dentista (ARUL ASKJ, et al., 2012; MORAIS EF, et al., 2014). Dessa forma, as manifestações orais podem atuar como um indicador diagnóstico nas leucemias. Para isso, o cirurgião-dentista necessita conhecer as manifestações orais comuns na leucemia para que possa intervir na saúde bucal do paciente, contribuindo e auxiliando no seu tratamento (ASHOK L, et al., 2010; MORAIS EF, et al., 2014).

A maioria dos artigos utilizados nesta revisão analisaram participantes com Leucemia linfoide. Quatro destes estudos analisaram uma amostra mista, pacientes com leucemia linfóide e leucemia mielóide, e apenas Shankarapillai $R$, et al. (2010) utilizaram em seu estudo apenas pacientes com leucemia mielóide.

Apenas dois estudos avaliaram pacientes com leucemia crônica, os demais estudaram apenas leucemia aguda. Todos identificaram lesões orais nos participantes leucêmicos. A Leucemia linfóide aguda é o tipo mais comum de leucemia (ARUL ASKJ, et al., 2012), sendo o tipo de câncer mais comum em crianças (COSTA SS, et al., 2011), o que justifica a maioria dos estudos utilizarem amostra com esse tipo de leucemia.

Ainda se sabe que a fisiopatologia da leucemia se explica pela malignização das células-tronco hematopoiéticas (CTH), que são células que possuem a capacidade de se autorrenovar e se diferenciar em células especializadas do sangue ou do sistema imune (SILVA-JÚNIOR FC, et al., 2009). As CTH geram células progenitoras multipotentes que vão dar origem aos progenitores comuns das linhagens mielóide e linfóide.

As células da linhagem mielóide são eritrócitos, plaquetas, macrófagos, neutrófilos, eosinófilos, basófilos, e as da linhagem linfoide, por sua vez, são os linfócitos B e T e células NK (SILVA-JÚNIOR FC, et al., 2009; AROSA FA, et al., 2012). Sendo assim, as características histológicas do tecido infiltrado são similares para todos os subtipos de leucemia, variando apenas a natureza da célula invasora, de acordo com o tipo de leucemia (CARNEIRO FM, et al., 2008).

A cavidade oral, e especialmente o tecido gengival, é um local comumente envolvido por infiltração de células leucêmicas devido à sua microanatomia e expressão de moléculas de adesão endotelial (PATIL S, et al., 2010). Segundo ARUL ASKJ, et al. (2012) a infiltração gengival leucêmica é mais comum na leucemia aguda. Dessa forma, as manifestações bucais das leucemias são mais comuns na fase aguda da doença, principalmente na forma linfóide, do que nas formas crônicas (PINTO MTF, et al., 2013).

Além disso, nas leucemias agudas, as células leucêmicas não podem fazer nenhum trabalho das células sanguíneas normais, perdendo sua atividade rapidamente. Porém, nas leucemias crônicas os sinais clínicos aparecem gradualmente com 0 agravamento da doença e, inicialmente, as células leucêmicas ainda conseguem fazer algum trabalho dos glóbulos brancos normais (FRANCISCONI FC, et al., 2016; SANTOS MMF, et al., 2019). Assim, a cavidade oral fica mais sujeita às complicações que variam desde lesões de mucosa e infecções até a exacerbação de condições patológicas preexistentes, colocando a vida do paciente em risco (CARNEIRO FM, et al, 2008).

Sabe-se que as leucemias agudas são neoplasias primárias da Medula Óssea, diagnosticadas tanto em adultos como crianças (FRANCISCONI FC, et al., 2016). Nas leucemias crônicas, por sua vez, acometem principalmente indivíduos adultos (SANTOS MMF, et al., 2019). Isto justifica o perfil das amostras nos estudos analisados, onde a maioria era composta por adolescentes e/ou crianças. Ainda se sabe que quanto mais jovem maior a probabilidade de a terapia antineoplásica afetar a cavidade oral (PINTO MTF, et al., 2013). Este fato pode estar relacionado ao índice mitótico elevado das células da mucosa bucal em crianças abaixo dos 12 anos de idade. 
Sobre a modalidade de tratamento, se sabe que as principais alternativas para 0 tratamento das neoplasias malignas são a cirurgia, radioterapia e quimioterapia, sendo, na maioria dos casos, necessário a combinação entre diferentes técnicas (BRANDÃO HN, et al., 2010). Dentre as modalidades de tratamento, a quimioterapia é a que possui maior índice de cura e a que mais aumenta a sobrevida dos pacientes oncóticos (BONASSA EMA e GATO MIR, 2012).

No entanto, os agentes quimioterápicos atuam nas células em proliferação sem distinguir as células neoplásicas das normais, o que faz a mucosa bucal ser um dos alvos dessa terapia (HESPANHOL FL, et al., 2010). Contudo, apesar de a maioria dos artigos que fazem parte do estudo citarem as drogas ou protocolos de tratamento utilizadas durante a quimioterapia, não foi possível estabelecer nesta revisão uma relação entre quimioterápico, dose e manifestação bucal, já que nos estudados não havia esse tipo de informação.

Segundo Jesus LG, et al. (2016), o efeito citotóxico dos quimioterápicos pode promover uma ação direta ou indireta sobre a mucosa oral. No primeiro caso, as drogas interferem sobre a proliferação celular, provocando a redução da renovação das células do epitélio e, consequentemente, descamação, ulceração, inflamação e atrofia.

Por outro lado, o efeito indireto está associado à ação dos agentes quimioterápicos sobre a medula óssea, o que leva a mielossupressão (interrupção da produção dos elementos figurados do sangue), resultando em anemia, trombocitopenia, plaquetopenia e leucopenia, propiciando o surgimento de hemorragias e infecções oportunistas (MARTINS ACM, et al., 2002; BONASSA EMA e GATO MIR, 2012; JESUS LG, et al., 2016).

Apesar de diversos trabalhos na literatura mostrarem uma correlação entre os tratamentos oncológicos e as lesões orais, é importante ressaltar que a gravidade das lesões depende de uma série de outros fatores relacionados ao tratamento, ao tumor e ao paciente (idade, estado nutricional, alterações na produção salivar, o trauma local e o nível de higiene bucal, antes e durante a terapia antineoplásica) (HESPANHOL FL, et al., 2010; LOPES IA, et al., 2012). Também se sabe que o comprometimento da cavidade bucal pode surgir no estágio inicial da leucemia, aumentando sua intensidade com a indução da mielossupressão pela terapia antineoplásica (FRANCISCONI FC, et al., 2016; GAZZINELLI LB, et al., 2018).

Segundo Pels EJ, et al. (2017), parâmetros sanguíneos influenciam o nível de comprometimento da mucosa oral. Pois ao avaliar a cavidade oral de crianças com leucemia linfoblástica aguda esses autores verificaram que as lesões eram dependentes do nível de neutropenia e que a cura das lesões foi mais rápida, principalmente em relação à ulceração da mucosa oral, quando os parâmetros morfológicos do sangue foram melhorados. Isso demonstra a importância das características hematológica para a saúde bucal do paciente. No entanto, poucos estudos citaram a verificação ou correlação das condições sanguíneas do paciente com as manifestações clínicas observadas. Para Coronel JP, et al. (2016), é fundamental que estudos sejam realizados para comprovar a relação da saúde periodontal com os parâmetros hematológicos.

As manifestações orais registradas nos estudos em participantes com leucemias foram mucosite, gengivite, sangramento gengival, hiperplasia gengival, úlceras na mucosa, petéquias, equimoses, palidez da mucosa, alteração da microbiota oral, despapilação lingual, deterioração da saúde bucal, aumento da experiência de cárie, redução do $\mathrm{pH}$, imunoglobulinas e antioxidantes salivares, xerostomia e distúrbio no desenvolvimento dentário e radicular. Segundo Carneiro FM, et al. (2008), de acordo com o agente causal, estes sinais e sintomas podem ser classificadas em primárias, secundárias e terciárias.

A infiltração celular e alargamento do tecido gengival são complicações classificadas como primárias, uma vez que são, normalmente, resultantes da infiltração direta dos tecidos bucais pelas células leucêmicas ou por hiperplasia inflamatória reacional (ARUL ASKJ, et al., 2012; FRANCISCONI FC, et al., 2016).

Já as ulcerações, sangramentos e palidez da mucosa estão relacionadas à citotoxidade indireta em decorrência do quadro anêmico, plaquetopenia e leucopenia, que pode levar a um atraso na cicatrização de feridas e palidez da mucosa bucal (CARNEIRO FM, et al., 2008). A redução de função e contagem normal dos leucócitos também resultam no aumento da susceptibilidade da cavidade oral a infecções, uma vez que estas células são responsáveis pela proteção do organismo (JESUS LG, et al., 2016). 
Por último, as complicações terciárias são explicadas pela terapia adotada e incluem efeitos de citotoxicidade direta e indireta. Neste caso, a mucosite, xerostomia, neurotoxidade, desordens temporomandibulares, alterações na estrutura e erupção dentária e sangramentos orais são complicações frequentes em pacientes submetidos à terapia antileucêmica (CARNEIRO FM, et al., 2008; JESUS LG, et al., 2016).

Os efeitos adversos do tratamento antineoplásico à cavidade oral podem se manifestar tanto de forma aguda (durante o tratamento) como a longo prazo (pós-tratamento) (MORAIS EF, et al., 2014; JESUS LG, et al., 2016). Vale ressaltar, que em alguns pacientes as manifestações orais podem se apresentar sintomas antes do tratamento quimioterápico e podem se apresentar como evidência inicial da doença ou de sua recidiva, sendo, portanto, o resultado da invasão da medula óssea e substituição das células saudáveis na mucosa oral pelas células neoplásicas (CARNEIRO FM, et al., 2008; ASHOK L, et al., 2010; FRANCISCONI FC, et al., 2016).

Sendo assim, lesões orais são comuns em pacientes leucêmicos tanto pela fisiologia da doença como pelos agentes antineoplásicos utilizados no tratamento, estes últimos exacerbam o surgimento de tais lesões (HEGDE AM, et al., 2011; JOSHI S, et al., 2013). Além disso, as complicações orais podem ser agravadas com a higiene oral precária, uma vez que a imunossupressão e lesões na mucosa oral são comuns em pacientes leucêmicos (TRINDADE AKF, et al., 2009; OLIVEIRA MC, 2016).

Portanto, é importante manter uma boa higiene oral nesses pacientes. Neste sentido, torna-se essencial a incorporação do cirurgião-dentista na equipe multidisciplinar que cuida da saúde do paciente oncótico. Esse profissional pode atuar tanto no reconhecimento das lesões, auxiliando no diagnóstico da doença, como no tratamento dos problemas orais, anteriores e/ou posteriores ao tratamento antineoplásico, para evitar e prevenir complicações (MORAIS EF, et al., 2014). Pois estudos comprovam que a aplicação de medidas preventivas bucodentais ajudou a evitar complicacões das lesões bucais encontradas em pacientes leucêmicos (CORONEL JP, et al., 2016).

\section{CONSIDERAÇÕES FINAIS}

Pacientes com leucemia normalmente apresentam alterações na mucosa oral. Mucosite, gengivite, sangramento e hiperplasia gengival, úlceras na mucosa, petéquias, equimoses, palidez da mucosa, alteração da microbiota oral, despapilação lingual, deterioração da saúde bucal, problemas periodontais, aumento da experiência de cárie, redução do $\mathrm{pH}$ e imunoglobulinas salivar, redução dos níveis de antioxidantes salivares, xerostomia, erupção tardia, distúrbio no desenvolvimento dentário e radicular são alterações orais que podem surgir na mucosa oral de leucêmicos. A ocorrência, gravidade e tipo de sinais e sintomas variam de indivíduo para indivíduo e dependem do tipo de leucemia, do tratamento, das condições hematológicas, idade e o nível de higiene oral do paciente.

\section{AGRADECIMENTOS}

À coordenação do curso de graduação em odontologia da Faculdade de Integração do Sertão que possibilitou a realização do estudo.

\section{REFERÊNCIAS}

1. ANGST PDM, et al. Gingival inflammation and platelet count in patients with leukemia: preliminary results. Brazilian Oral Research, 2011; 25(6): 544-549.

2. AROSA FA, et al. 2nd Ed. Fundamentos de Imunologia. Lidel-Edições Técnicas: Lisboa, 2012; 640p.

3. ARUL ASKJ, et al. A clinical and fine needle aspiration cytology study of gingiva in acute leukemia. Dental Research Journal, 2012; 9(1): 80-85.

4. ASHOK L, et al. Estimativa de amilase salivar e proteínas totais em pacientes com leucemia e sua correlação com características clínicas e achados radiográficos. Indian Journal Dental Research, 2010; 21(4): 486-490.

5. AZHER U, SHIGGAON N. Oral health status of children with acute lymphoblastic leukemia undergoing chemotherapy. Indian Journal Dental Research, 2013; 24(4): 523-526.

6. BONASSA EMA, GATO MIR. Terapêutica oncológica para enfermeiros e farmacêuticos. 4nd ed. São Paulo: Atheneu, 2012; 650p. 


\section{Revista Eletrônica Acervo Saúde / Electronic Journal Collection Health | ISSN 2178-2091}

7. BRANDÃO HN, et al. M. Química e farmacologia de quimioterápicos antineoplásicos derivados de plantas. Química Nova, 2010; 33(6): 1359-1369.

8. CARNEIRO FM, et al. Manifestações bucais das leucemias agudas na infância. Arquivo Brasileiro de Odontologia, 2008; 4(1): 40-54.

9. CORONEL JP, et al. Condiciones de salud bucodental en niños menores de catorce años con leucemia linfoblástica aguda, antes del tratamiento de quimioterapia en el Instituto del Cáncer (SOLCA), Cuenca. Revista de Odontologia da Universidade de São Paulo, 2016; 28(3): 194-200.

10. COSTA SS, et al. Conhecimento de manifestações orais da leucemia e protocolo de atendimento odontológico. Revista de Odontologia da Universidade de São Paulo, 2011; 23(1): 70-78.

11. FINOTTI ACF, et al. Epidemiologia do transplante de medula óssea no Hospital Araújo Jorge de 2000 a 2004 em Goiânia, Goiás. Estudos de Biologia, 2006; 28(65): 77-85.

12. FRANCISCONI FC, et al. Leukemic oral manifestations and their management. Asian Pacifis Journal of Cancer Prevention, 2015; 17(3): 911- 915.

13. GAZZINELLI LB, et al. Manejo odontológico em crianças com leucemia aguda sob tratamento antineoplásico. Revista Uningá, 218;55(1): 121-133.

14. GHOLIZADEH N, et al. Palifermin and chlorhexidine mouthwashes in prevention of chemotherapyinduced mucositis in childrenwith acute lymphocytic leukemia: a randomized controlled trial.Journal of Dentistry(Shiraz Universityof Medical Sciences, 2016; 17(4): 343-347.

15. GRAZIELE C, et al. Diagnóstico laboratorial das leucemias mielóides aguda. Jornal Brasileiro de Patologia e Medicina Laboratorial, 2006; 42(2): 77-84.

16. HEGDE AM, et al. Evaluation of oral hygiene status, salivary characteristics and dental caries experience in acute lymphoblastic leukemic (ALL) children. Journal of Clinical Pediatric Dentistry, 2011; 35(3): 319-323.

17. HESPANHOL FL, et al. Manifestações bucais em pacientes submetidos à quimioterapia. Ciência \& Saúde Coletiva, 2010; 15(1): 1085-1094.

18. JAVED F, et al. Oral health status in children with acute lymphoblastic leukemia. Critical Reviews Oncology/Hematology, 2012; 83(3): 303-309.

19. JESUS LG, et al. Repercussões orais de drogas antineoplásicas: uma revisão de literatura. Revista da Faculdade de Odontologia-UFPE, 2016; 21(1): 130-135.

20. JOSHI S, et al. Evaluation of salivary sialic acid levels in acute lymphoblastic leukemic children and its correlation with dental caries experience. Journal of Clinical Pediatric Dentistry, 2013; 37(3): 309-313.

21. JUÁREZ-LÓPEZ MLA, et al. Oral diseases in children with acute lymphoblastic leukemia with chemotherapy treatment. Revista Médica del Instituto Mexicano del Seguro Social, 2018; 56(2):132-135.

22. LOPES IA, et al. Manifestações orais decorrentes da quimioterapia em crianças de um centro de tratamento oncológico. Pesquisa Brasileira em Odontopediatria e Clínica Integrada,2012;12(1): 113-119.

23. MARTINS ACM, et al. Complicações bucais da quimioterapia antineoplásica. Acta Scientiarum, 2002; 24(3): 663-670.

24. MCCORD C, JOHNSON L. Oral manifestations of hematologic disease. In: NAPEÑAS, J. J. Atlas of the oral and maxillofacial surgery clinics of North American. 1를. Ed. Pensennsylvania: Elsevier Health Sciences. 2017; 25: 149-162.

25. MORAIS EF, et al. Oral manifestations resulting from chemotherapy in children with acute lymphoblastic leukemia. Baz. J Otorhinolaryngol, 2014; 80(1): 78-85.

26. NELSON RW, COUTO CG. Medicina Interna de Pequenos Animais. 5ª Ed. São Paulo: GEN Guanabara Koogan, 2015; $1512 \mathrm{p}$.

27. OLIVEIRA MC, et al. Oral manifestations in pediatric patients receiving chemotherapy for leucemia. Stomatos, 2016: 22(43):20-30.

28. PATIL S, et al. Leukemic gingival enlargement: a report of two cases. Archives of Orofacial Sciences, 2010; 5(2): 69-72.

29. PELS EJ. Oral mucositis and saliva $\lg A$, $\lg G$ and $\lg M$ concentration during anti-tumor treatment in children suffering from acute lymphoblastic leukemia. Advances in Clinical and Experimental Medicine, 2017; 26(9): 1351-1358.

30. PINTO MTF, et al. Prevalência de manifestações orais em pacientes infanto-juvenis submetidos à quimioterapia. Revista de Pesquisa em Saúde, 2013; 14(1): 45-48.

31. PONCE-TORRES E, et al. Oral manifestations in pediatric patients receiving chemotherapy for acute lymphoblastic leukemia.Journal of Clinical Pediatric Dentistry, 2010; 34(3):275-279.

32. SANTOS MMF, et al. Leucemia mieloide, aguda e crônica: diagnósticos e possíveis tratamentos. Revista Saúde em Foco, 2019; 11: 279-294.

33. SHANKARAPILLAI R, et al. Periodontal and gingival parameters in young adults with acute myeloid leukemia in Kerala, South India. Oral Health and Preventive Dentistry, 2010; 8(4): 385-400.

34. SILVA-JÚNIOR FC, et al. Células-tronco hematopoéticas: utilidades e perspectivas. Revista Brasileira de Hematologia e Hemoterapia, 2009; 31(1): 53- 58.

35. SOARES AF, et al. Frequency of oral mucositis and microbiological analysis in children with acute lymphoblastic leukemia treated with 0.12\% chlorhexidine gluconate. Brazilian Dental Journal, 2011; 22(4): 312-316.

36. THOMAZ EBA, et al. Longitudinal assessment of immunological and oral clinical conditions in patients undergoing anticancer treatment for leukemia. International Journalof Pediatric Otorhinolaryngology,2013; 77(7): 1088-1093.

37. TRINDADE AKF et al. Manifestações orais em pacientes pediátricos leucêmicos. Arquivos em Odontologia, 2009; 45(1): 22-29.

38. VALÉRA MC, et al. Oral complications and dental care in children with acute lymphoblastic leukaemia. Journal of Oral Pathology \& Medicine, 2014; 44(7): 483-489.

39. VASCONCELOS NPS, et al. Dental maturity assesmentin children with acute lymphoblastic leucemia after cancer therapy. Forensic Science International, 184(1-3): 10-14.

40. WANG Y, et al. Oral microbiota distinguishes acute lymphoblastic leukemia pediatric hosts from healthy populations. Plos One, 2014; 9(7): 1-8. 